\title{
DISCUSSÃO GEOMÉTRICA PARA EQUAÇÕES QUADRÁTICAS E ELEMENTOS DE ENGENHARIA DIDÁTICA: ANÁLISES PRELIMINARES E A PRIORI
}

\author{
Guttenberg Sergistótanes Santos Ferreira, Francisco Regis Vieira Alves \\ Instituto Federal de Educação, Ciência e Tecnologia do Ceará (IFCE) \\ Campus de Juazeiro do Norte \\ Programa de Pós-graduação em Ensino de Ciências e Matemática (PPGER) \\ Campus de Fortaleza \\ <guttenberg@ifce.edu.br>, <fregis@ifce.edu.br>
}

DOI: 10.21439/conexoes.conexoes.v10i4.1102

\begin{abstract}
Resumo. A resolução de Equações Quadráticas, quando discutida na Educação Básica assim como no Ensino Superior, muitas vezes é feita apenas de forma algébrica. Neste artigo discutimos uma metodologia para resolução de equações quadráticas com viés geométrico, através do Método de Descartes e do Método das Semicircunferências Tangentes. Estes modelos tratam da localização dos zeros de equações quadráticas através de elementos da Geometria Plana. Desta forma, este trabalho objetivou realizar um estudo contextualizado sobre equações quadráticas apoiado nos pressupostos da Engenharia Didática, descrevendo apenas as etapas iniciais: análises preliminares, e construção e análises a priori. A descrição das etapas iniciais da Engenharia Didática prevê explorar os aspectos investigativos no estudante possibilitando a experimentação matemática através de situações didáticas de ensino, inclusive com uso recursos tecnológicos utilizando o software GeoGebra.
\end{abstract}

Palavras-chaves: Ensino de Matemática. Equações Quadráticas. Engenharia Didática. Interpretação Geométrica.

Abstract. The resolution of Quadratic Equations, when discussed in Basic Education as well as in Higher Education, is often only done in an algebraic way. In this paper we discuss a methodology for the resolution of quadratic equations with geometric bias, through the Descartes Method and the Tangent Semicircunference Method. These models deal with the location of zeros of quadratic equations through elements of Flat Geometry. In this way, this work aimed to perform a contextualized study on quadratic equations based on the presuppositions of Didactic Engineering, describing only the initial stages: preliminary analysis, and construction and analysis a priori. The description of the initial stages of Didactic Engineering intends to explore the investigative aspects in the student allowing the mathematical experimentation through teaching didactic situations, including using technological resources using GeoGebra software.

Keywords: Mathematics Teaching. Quadratic equations. Didactic Engineering. Geometrical interpretation. 


\section{INTRODUÇÃO}

A resolução de equações quadráticas, também conhecidas como equações polinomiais de $2^{\circ}$ grau, quando discutida nos livros de Matemática ou de História da Matemática, remete sua contextualização à figura do matemático hindu Bhaskara (1114 - 1185), mesmo existindo relatos que os babilônios foram uma das primeiras civilizações a desenvolverem estudos e aplicações sobre tais equações. De acordo com Contador (2008, p.80), "fontes babilônicas antigas revelam a presença de equações do segundo grau e tudo indica que sua origem esteja relacionada com a vontade dos babilônios em querer saber qual a relação entre o perímetro e a área de um retângulo".

Apresentamos abaixo uma situação matemática que é uma das precursoras no desenvolvimento das equações quadráticas. Assim, seja o retângulo representado pela Figura 1, em que seu perímetro $(2 p)$ seja fornecido pela equação $2 p=2 x+2 y$, com isso $p=x+y$; e sua área pode ser calculada como sendo $q=x \cdot y$. Assumindo que $y=\frac{q}{x}$, temos que $x+\frac{q}{x}=p$, e $x^{2}-p x+q=0$.

Situações semelhantes à discutida acima propiciaram o desenvolvimento sistemático do raciocínio abordado, denotando situações reais para resolução de problemas (FERREIRA; ALVES, 2015). A equação ou fórmula resolutiva para equações quadráticas é bastante conhecida em literatura especializada como fórmula de Bhaskara, mas o próprio Bhaskara afirmou por volta do séc. XII que esta descoberta se devia a Sridhara (matemático hindu, 870 - 930) (GARBI, 2010). Na próxima seção traremos ao leitor uma discussão sobre a Fórmula de Bhaskara e a relação com suas raízes. E em seguida, apresentaremos os modelos matemáticos para localização das raízes de equações quadráticas a fim de estruturarmos o desenvolvimento do raciocínio geométrico para resolução de tais equações, sendo estes os objetivos deste estudo, ratificando que a proposta deste trabalho é de subsidiar estudos sobre alternativas metodológicas de resolução de equações quadráticas, sobretudo nos aspectos geométricos.

\section{1 modelo de Bhaskara}

O modelo algébrico com o qual se discute a fórmula de Bhaskara está embasado na expressão polinomial $P(x)=a_{0}+a_{1} x+a_{2} x^{2}+\cdots+a_{n-1} x^{n-1}+a_{n} x^{n}$, esta generalização é reduzida para uso de polinômios de $2^{\circ}$ grau, de modo que são reconhecidas as equações quadráticas como: $a x^{2}+b x+c=0(a \neq 0)$. Recorrendo aos ensinamentos de Euclides e desenvolvendo a equação quadrática genérica acima, temos que $x^{2}+\frac{b}{a} x+\frac{c}{a}=0$, donde $x^{2}+\frac{b}{a} x+\frac{b^{2}}{4 a^{2}}=-\frac{c}{a}+\frac{b^{2}}{4 a^{2}}$. Segue que $\left(x+\frac{b}{2 a}\right)^{2}=\frac{b^{2}-4 a c}{4 a^{2}}, \log 0 x=-\frac{b}{2 a} \pm$ $\sqrt{\frac{b^{2}-4 a c}{4 a^{2}}}$, que indica a equação resolutiva como sendo $x=\frac{-b \pm \sqrt{b^{2}-4 a c}}{2 a}$.

A construção/dedução da equação acima possibilitou a localização exata das raízes, sendo distintas ou não, mas também indicou a qual conjunto numérico esses zeros pertenciam, se ao conjunto dos números reais ou dos números complexos. Isto se deu devido ao uso da expressão b2-4ac, que recebeu o nome de Discriminante e é denotado pela letra grega $\Delta$ (delta maiúscula), e possui como função inicial a determinação do conjunto numérico à qual essas raízes estão vinculadas (FERREIRA, 2016). Contribuindo com essa discussão sobre resolução de equações quadráticas, indicamos também o estudo com viés geométrico, apresentando a seguir alguns modelos que tratam dessa ressignificação da fórmula de Bhaskara.

Na próxima seção faremos uma discussão sobre o modelo geométrico desenvolvido pelo matemático e filósofo francês Descartes (1596 - 1650) para resolução de equações quadráticas. Este método apresenta a localização das duas raízes reais, dadas em módulo, pois à época não eram reconhecidas as raízes negativas.

\section{ESTUDO DOS MÉTODOS GEOMÉTRICOS}

\subsection{Método de Descartes - MD}

O modelo conhecido como Método de Descartes (matemático francês, 1596 - 1650) pode ser utilizado para resolução de equações do tipo $x^{2} \pm b x-c=0$ e indica a elaboração/construção de um triângulo retângulo, de catetos $\frac{b}{2}$ e $\sqrt{c}$, e de uma circunferência cujo raio seja igual ao cateto menor do triângulo retângulo. Dessa forma, com essa construção, conseguimos não somente determinar as raízes da equação quadrática, mas também fazer uma releitura da fórmula de Bhaskara. O estudo da Figura 2, aliado ao tipo de equação que este modelo geométrico pode ser empregado, sugere-nos duas possibilidades: $b<0$ e $b>0$ (KILHIAN, 2012).

Para o caso em que $\mathrm{b}<0$ a raiz positiva é determinada pelo segmento $\overline{B D}$, enquanto que a raiz negativa é determinada pelo segmento $\overline{B E}$ (obviamente dada em módulo). Desta forma, denotando o segmento $\overline{B D}=x$, temos que $\overline{B D}=\overline{B E}+\overline{E C}+\overline{C D}, \log$ o $x=$ $y+\frac{b}{2}+\frac{b}{2}$, conforme Figura 3 de modo que $\overline{B C}=x-\frac{b}{2}$. Com estas informações e ainda utilizando o teorema de Pitágoras temos que $(\overline{B C})^{2}=(\overline{A C})^{2}+(\overline{A B})^{2}$, que implica que $\left(x-\frac{b}{2}\right)^{2}=\left(\frac{b}{2}\right)^{2}+(\sqrt{c})^{2}, \log 0 x^{2}-b x-c=0$.

Analogamente, discutimos o caso em que $b>0$. Temos que a raiz positiva é determinada pelo segmento 


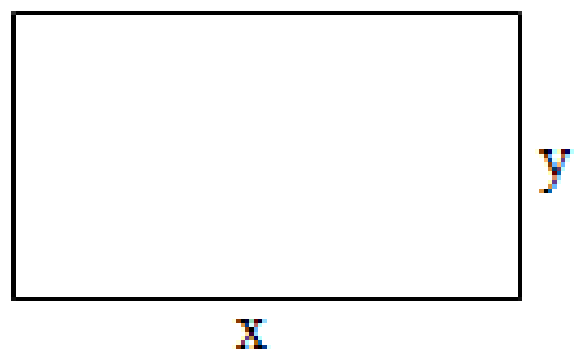

Figura 1: Retângulo de lados x e y.

Fonte: Formatação própria.

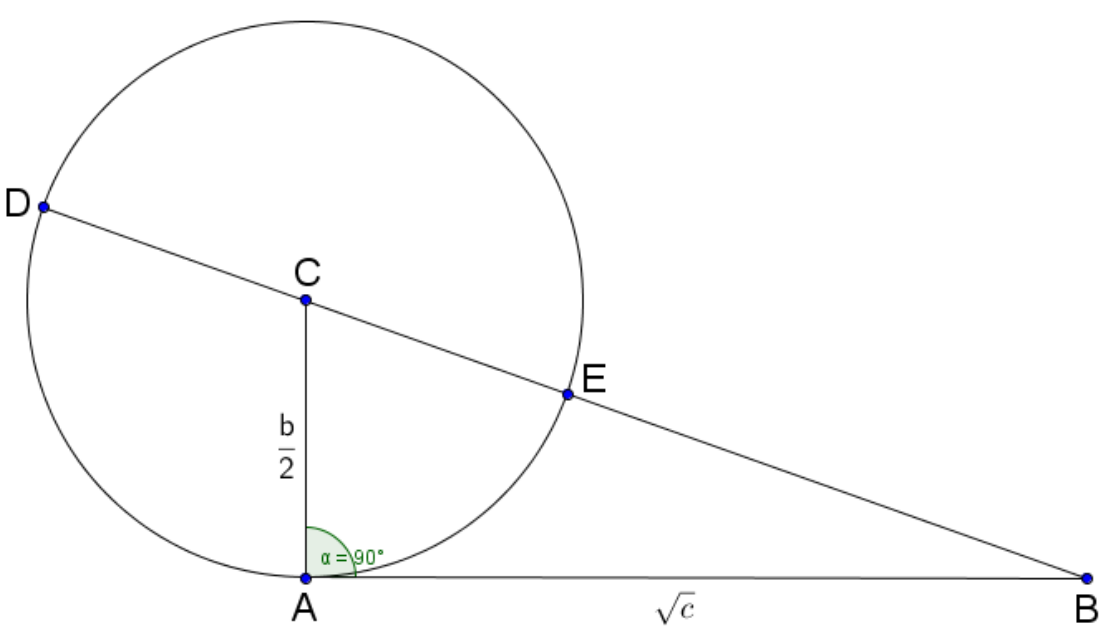

Figura 2: Circunferência de Descartes.

Fonte: Formatação própria baseada em Kilhian (2012). 


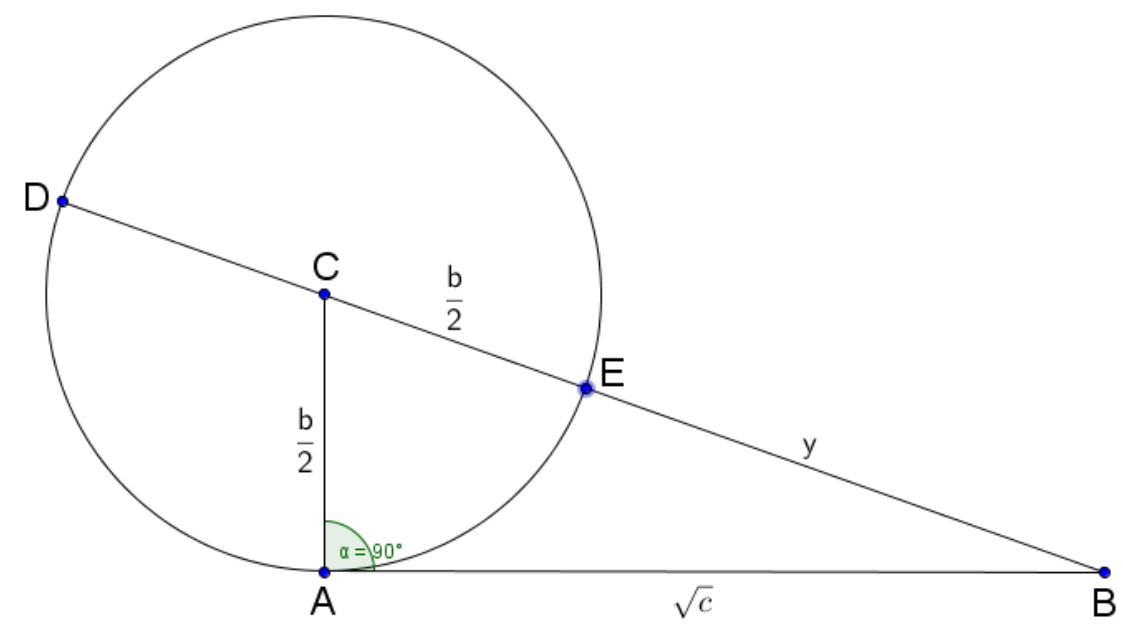

Figura 3: Circunferência de Descartes, caso $b<0$.

Fonte: Formatação própria baseada em Kilhian (2012).

$\overline{B E}$, enquanto que a raiz negativa é determinada pelo segmento $\overline{B D}$ (em módulo). De modo que ao denotarmos o segmento $\overline{B E}=x$, temos que $\overline{B C}=$ $\overline{B E}+\overline{E C}=x+\frac{b}{2}$, conforme Figura 4 . Mais uma vez utilizando o teorema de Pitágoras temos que $(\overline{B C})^{2}=(\overline{A C})^{2}+(\overline{A B})^{2}$, que implica que $\left(x+\frac{b}{2}\right)^{2}=$ $\left(\frac{b}{2}\right)^{2}+(\sqrt{c})^{2}, \log 0 x^{2}+b x-c=0$.

A visualização gráfica apresentada certamente incide de forma positiva quando do uso do modelo geométrico, propiciando ao estudante a experimentação laboratorial nem sempre acessível na disciplina de Matemática. Ressalte-se que o MD só foi concebido para equações quadráticas em que $c<0$. Desta forma, existe toda uma infinidade de equações que ainda necessitam de experimentação geométrica, aquelas em que $c>0$. A seguir, apresentaremos o Método das Semicircunferências Tangentes para resolução de equações quadráticas do tipo $x^{2} \pm b x \pm c=0$.

\subsection{Método das Semicircunferências Tangentes - MST}

O modelo geométrico conhecido como Método das Semicircunferências Tangentes também é utilizado na resolução/reinterpretação das equações quadráticas. Esta técnica surge com a associação de duas semicircunferências tangentes entre si e retas perpendiculares de apoio. Este modelo se subdivide em dois casos: $c<0$ e $c>0$ (TUNALA, 1988).
Para o caso em que $c>0$ temos que as raízes possuem mesmo sinal, e ainda que $\left|x_{1}\right|+\left|x_{2}\right|=|b| \mathrm{e}$ $\left|x_{1}\right| \cdot\left|x_{2}\right|=c$, em que $x_{1}$ e $x_{2}$ denotam as raízes procuradas. Procedemos traçando segmentos consecutivos de comprimentos $c$, 1 e $|b|$, conforme Figura 5, e percebemos ainda, através de relações métricas no triângulo retângulo, que $(\overline{G J})^{2}=\overline{F G} \cdot \overline{G H} \operatorname{logo} \overline{G J}=\sqrt{c}$. Sabendo que $\overline{G J}=\overline{G J}$ e $(\overline{M L})^{2}=\overline{H M} \cdot \overline{M I}$, obtemos que $\overline{H M} \cdot \overline{M I}=c$ e $\overline{H M}+\overline{M I}=|b|$. De onde podemos concluir que as raízes procuradas são numericamente iguais aos segmentos $\overline{H M}$ e $\overline{M I}$ para $b<0$, ou ainda $-\overline{H M} \mathrm{e}-\overline{M I}$ para $b>0$.

Para o caso em que $c<0$ temos que as raízes possuem sinais distintos e que $\left|x_{1}\right|-\left|x_{2}\right|=|b| \mathrm{e}$ $\left|x_{1}\right| \cdot\left|x_{2}\right|=c$ conforme Figura 6 . Trabalhando de modo análogo ao caso $c>0$, procedemos à montagem do modelo sendo $\overline{H M}=\overline{M N}=\overline{P M}$ e $\overline{L M}=\overline{L P}+\overline{P M}$, segue que $(\overline{L M})^{2}=(\overline{H L})^{2}+(\overline{H M})^{2}$ cujo desenvolvimento binomial nos fornece $\overline{L P} \cdot \overline{L N}=(\overline{H L})$, que nos permite concluir que $\overline{L P}$ e $\overline{L N}$ são os zeros procurados. Vale destacar que se $b<0$ então as raízes são $\overline{L N}$ e $-\overline{L P}$, enquanto que se $b>0$ então as raízes são determinadas por $-\overline{L N}$ e $\overline{L P}$.

A técnica acima apresentada proporciona ao leitor uma releitura das equações quadráticas trazendo uma abordagem geométrica bastante semelhante ao modelo proposto por Descartes, porém de forma mais completa. Apesar de ser um tanto mais complexo e de possuir maior raio de abrangência, o MST é proposto como ex- 


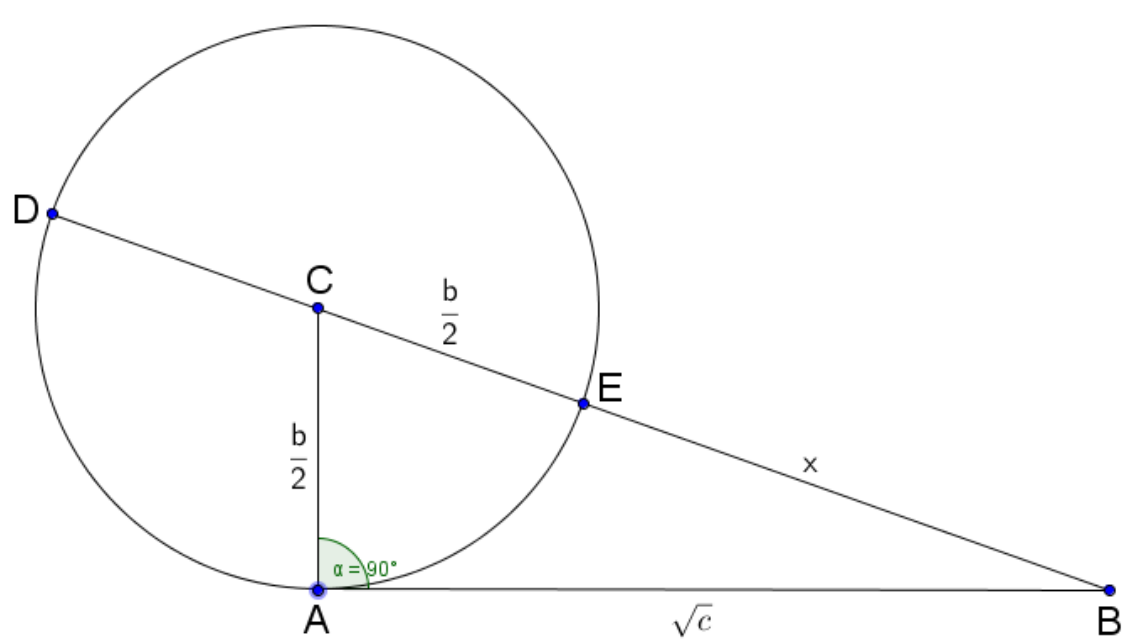

Figura 4: Circunferência de Descartes, $c a s o b>0$.

Fonte: Formatação própria baseada em Kilhian 2012).

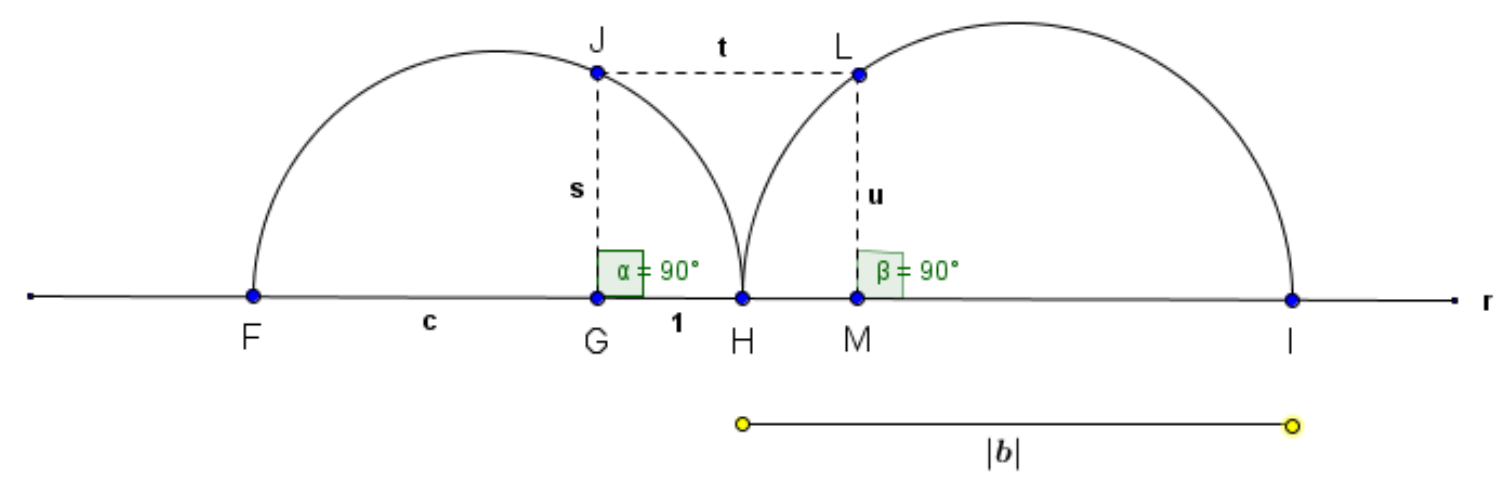

Figura 5: Semicircunferências tangentes, caso $c>0$.

Fonte: Formatação própria baseada em Kilhian (2012). 


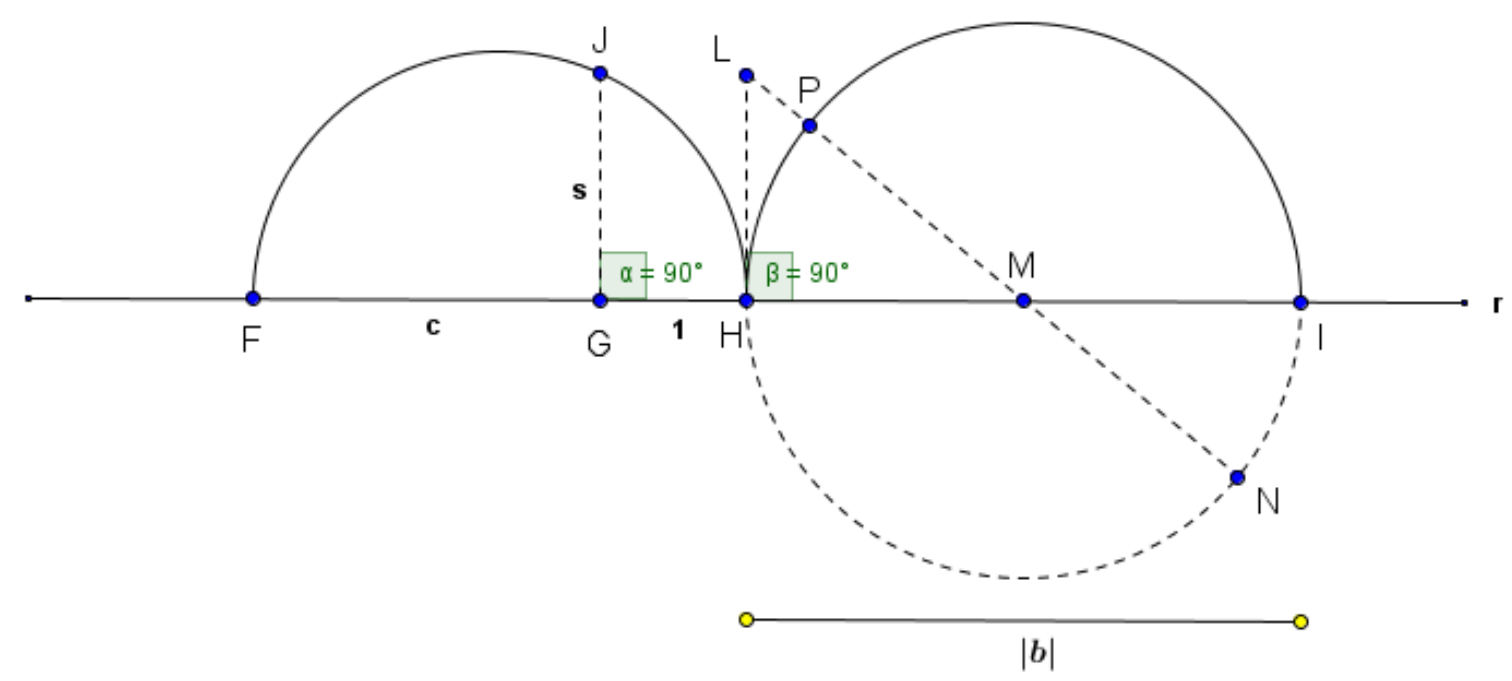

Figura 6: Semicircunferências tangentes, caso $c<0$.

Fonte: Formatação própria baseada em Tunala $(1988)$

perimentação laboratorial e ainda como uma revisão de diversos conceitos abordados na Geometria Plana.

Nas próximas seções faremos uma breve introdução/descrição sobre a metodologia de ensino e pesquisa conhecida como Engenharia Didática - ED; e ainda, discutiremos sobre as análises preliminares e análises a priori para interpretação geométrica da fórmula de Bhaskara, ressaltando seu grau de importância para o desenvolvimento sistematizado do ensino de Matemática segundo os pressupostos da ED.

\section{ENGENHARIA DIDÁTICA - ED}

A Engenharia Didática é uma tendência metodológica de ensino e pesquisa desenvolvida pela pesquisadora e educadora matemática Michelle Artigue, em meados da década de 1980, na escola francesa de Educação Matemática, mais especificamente no IREM - Instituto de Investigação em Ensino de Matemática (FERREIRA; ALVES, 2015). Esta teoria pode ser definida como uma variação metodológica do trabalho didático e que pode ser comparado ao trabalho realizado pelo engenheiro; este trabalho deve se apoiar em conhecimentos científicos, bem como deve ser submetido a normas de controle também científicos e ainda procurar trabalhar com objetos mais complexos (ALMOULOUD; QUEIROZ; COUTINHO, 2008).

A ED foi desenvolvida com ênfase na discussão de uma problemática com duas vertentes: das relações que existem entre pesquisa e sistemas de ensino; e também sobre o espaço destinado às práticas pedagógicas junto à metodologia da pesquisa. Para que haja o fomento e correlação direta da pesquisa em Matemática com a realidade do ensino de Matemática, é que surge a ED como alternativa possível, e pode ser utilizada constantemente, para sistematizar o processo de ensino, e fortalecer as relações de aprendizagem, trazendo reflexões aos professores e estudantes, enquanto discute práticas pedagógicas relacionando pesquisa e espaço destinado às mesmas (CARNEIRO, 2005).

A ED se fundamenta em cinco fases: Análises Preliminares; Construção e Análise a Priori; Experimentação; Análise a Posteriori e Validação. Neste estudo discutiremos somente as duas fases iniciais desta teoria: Análises Preliminares; Construção e Análise a Priori; aplicadas às interpretações geométricas da fórmula de Bhaskara já propostas: o Método de Descartes e o Método das Semicircunferências Tangentes.

\subsection{Análises Preliminares}

Nesta fase inicial da ED se espera que o docente em Matemática realize todo o planejamento necessário para que a atividade proposta aos discentes tenha êxito, considerando para isto os objetivos a serem alcançados e em quais condições de trabalho, quer possua dificulda- 
des físicas ou ainda comportamentais. Sobre isto, Almouloud (2007, p. 172) destaca dois elementos essenciais à discussão: estudo da organização matemática; e análise didática do objeto matemático escolhido.

Para discutirmos o estudo da organização matemática recorremos à origem do desenvolvimento da fórmula de Bhaskara para resolução de equações quadráticas, bem como de sua interpretação geométrica, ressalvados os casos em que $\Delta<0$, pois este estudo não se propõe, a discutir raízes imaginárias no Conjunto dos Números Complexos. A outra vertente a ser abordada se refere ao trabalho em sala de aula, especificamente no uso didático dos modelos matemáticos já descritos, vistos aqui não apenas como uma proposta de aula com experimentação laboratorial de Matemática, mas também com a intenção de ressignificar os saberes matemáticos e favorecer a discussão algébrico-geométrica. Com isso, não pretendemos discutir neste estudo o caráter abstracionista tão característico em Matemática, e sim propiciar ao estudante o contato com uma Matemática real e palpável, de modo que ele possa manipular seus elementos através da experimentação laboratorial, tão presente na pesquisa acadêmica. Posto isto, discutimos os possíveis obstáculos conceituais advindos deste estudo, uma vez que não basta apenas conhecer o desenvolvimento da fórmula de Bhaskara, ou ainda de seus elementos pertencentes à Geometria Plana, mas sim, em fazer correta associação a fim de reinterpretar os saberes aqui discutidos.

Ao se propor a discussão quanto à análise didática do objeto matemático, vamos observar a abordagem presente em livros de História da Matemática ou, de forma incipiente, nos apêndices históricos presentes nos livros de Matemática da Educação Básica, e ainda prever dificuldades cognitivas em detrimento da aprendizagem. Consultando diversas obras de Matemática (DANTE, 2004; SILVA; FILHO, 2005, GIOVANNI; BONJORNO; JUNIOR, 2005; MARCONDES; GENTIL; GRECO, 2003; PAIVA, 2005; SMOLE; DINIZ, 2005) ou de História da Matemática (BOYER; MERZBACH, 2010, CONTADOR, 2008; EVES; DOMINGUES 2004), não encontramos os modelos geométricos discutidos neste estudo, o que trouxe à reflexão sobre uma possível dificuldade dos estudantes em associar a abordagem geométrica, qualquer que seja, com a fórmula de Bhaskara, dá-se justamente devido à grande dos livros discutirem apenas a interpretação algébrica para equações quadráticas.

\subsection{Construção e Análises a Priori}

Nesta fase da Engenharia Didática se espera que o docente realize tanto a descrição quanto a análise da si- tuação adidática escolhida por ele e que será devidamente proposta ao estudante. Vale destacar que a situação adidática "é caracterizada pela oportunidade de sucesso do estudante, por meio de seus próprios méritos, que consegue sintetizar um conhecimento e empregálo de modo relativamente diferenciado da maneira pela qual foi ensinado" (ALVES, 2011(a), p. 77). Desta forma, ressaltamos papel do professor como mediador de aprendizagem, com a função de antever ações comportamentais que possam favorecer ou não o desenvolvimento do conhecimento lógico matemático aplicado na experimentação proposta, de modo que o professor aja concomitantemente com a dimensão cognitiva e epistemológica, além de fazer uso da didática em sala de aula (ALMOULOUD; QUEIROZ; COUTINHO, 2008).

A partir dos pressupostos sobre situação adidática afirmamos que a situação escolhida para este estudo sugere, de forma ampla, apenas a interpretação geométrica da fórmula de Bhaskara, que está intimamente ligada à correlação existente entre a área e o perímetro de um retângulo (GARBI, 2010). Contudo, uma abordagem mais específica se refere a aspectos conceituais no uso de circunferências e pontos de tangência para resolver a problemática aqui proposta, bem como a conceitos inerentes a triângulos retângulos. Sendo assim, o estudante não pode prescindir do conhecimento de Geometria Plana, a fim de evidenciar corretamente a interpretação geométrica do modelo de Bhaskara; e para experimentação laboratorial se sugere o uso e correta manipulação de software de geometria dinâmica GeoGebra, em que o estudante consegue não somente testar suas conjecturas mas também validar o conhecimento geométrico objeto deste estudo.

O estudante deve ainda compreender a problemática estudada a fim de desenvolver sua solução, ficando a cargo do professor propor uma tarefa passível de execução, enquanto media a aprendizagem e torna a situação adidática inicial num processo construtivista, ou seja, numa situação didática (ALVES, 2014). Desta forma, corroboramos que os objetivos dessa atividade baseada na ED são: auxiliar o estudante na construção e desenvolvimento do conhecimento, reinterpretar os saberes da fórmula de Bhaskara, inclusive com outras abordagens geométricas aliando a isto o uso do recurso computacional (ALMOULOUD, 2007).

A seguir, faremos uma discussão sobre as condições restritivas dos métodos geométricos já discutidos, que pode surgir quando da experimentação tecnológica feita pelo estudante. Dentre as situações possíveis nessa experimentação matemática, destacamos a discussão quanto à validade da teoria metodológica pro- 
posta, exemplificando para isto a condição quando o estudante deseja investigar/experimentar se os pressupostos restritivos são válidos ou não. Neste estudo, podemos apontar o caso em que $c>0$ para MD, ou $\Delta<0$ para MST, para isto usaremos três situações de estudo a fim de corroborar ou negar a eficiência dos métodos, ilustrando a experimentação discente.

Situação 1 É solicitada a resolução da equação quadrática $x^{2}-2 x-15=0$ e, posteriormente, sua construção geométrica através do MD. Em seguida, repetir o procedimento para a equação $x^{2}-3 x+2=0$ e por fim realizar um estudo comparativo entre as soluções.

Percebe-se que as soluções de ambas as equações podem ser obtidas com o uso da fórmula de Bhaskara. Sem maiores delongas, obtemos que as raízes da equação $x^{2}-2 x-15=0$ são dadas por $x_{1}=-3 \mathrm{e}$ $x_{2}=5$, e sua construção geométrica é apresentada na Figura 7 De modo análogo se procede com a equação $x^{2}-3 x+2=0$, cujas raízes são $x_{1}=1$ e $x_{2}=2$, e sua construção geométrica se encontra indicada ainda na Figura 7

Observamos que na equação $x^{2}-2 x-15=0$ o segmento $\overline{B E}=3$ coincide com a raiz $x_{1}$, porém dada em módulo. Entretanto, o mesmo não ocorre na equação $x^{2}-3 x+2=0$, em que $\overline{B E}=0,56$ diferindo da raiz $x_{1}=1$ da equação, apesar de ter sido submetido ao mesmo método construtivo, corroborando os aspectos restritivos, no caso $c>0$.

Ressaltamos que após realizar estas resoluções, o estudante deve ser estimulado a testar/experimentar outros formatos de equação segundo o $M D$, e com a mediação do professor corroborar ou refutar os casos em que $b>0$ e $c<0$, ou ainda, $b>0$ e $c>0$, considerando sempre a experimentação através da construção geométrica.

Situação 2 É solicitada a resolução da equação quadrática $x^{2}-4 x+3=0$ e, em seguida, a modelagem geométrica através do MST. Para fins de experimentação, sugere-se repetir o procedimento para a equação $x^{2}-4 x+5=0$ e por fim realizar um estudo comparativo entre as soluções.

Calculando as raízes da equação através da fórmula de Bhaskara, obtemos os zeros $x_{1}=1$ e $x_{2}=3$ para a equação $x^{2}-4 x+3=0$, cuja representação geométrica está apresentada na Figura 8 E, analogamente, para a equação $x^{2}-4 x+5=0$, cujas raízes são $x_{1}=\frac{4-3 \sqrt{-1}}{2}$ e $x_{2}=\frac{4+3 \sqrt{-1}}{2}$, representamos sua construção geométrica na Figura 9 .

Analisamos os segmentos $\overline{H M}=1$ e $\overline{M I}=3$ representam os zeros da equação $x^{2}-4 x+3=0$ (Figura
8), isto devido à interseção entre o segmento $\overline{J L}$ e o semicírculo com comprimento de raio igual a $4 \mathrm{e}$, posteriormente, à construção do segmento perpendicular $\overline{L M}$. Continuamos com a análise na equação $x^{2}-4 x+5=0$ (Figura 9), percebendo que não é possível a localização das raízes da equação, uma vez que não existe intersecção entre o segmento $\overline{J L}$ e o semicírculo com comprimento de raio igual a 4 denotando que as raízes da equação não pertencem ao Conjunto dos Números Reais, e sim, do Conjunto dos Números Complexos.

Situação 3 É solicitada a resolução da equação quadrática $x^{2}+2 x-3=0$ e, em seguida, a modelagem geométrica através do MST. Sugerimos, para fins de experimentação, a repetição do procedimento para a equação $x^{2}+3 x+3=0$ para realizar um estudo comparativo entre as soluções.

Sobre as raízes da equação, calculadas por intermédio da fórmula de Bhaskara, obtemos os zeros $x_{1}=-3$ e $x_{2}=1$ para a equação $x^{2}+2 x-3=0$, representada geometricamente na Figura 10 . E, analogamente, para a equação $x^{2}+3 x+3=0$, cujas raízes são $x_{1}=\frac{-3-\sqrt{-3}}{2}$ e $x_{2}=\frac{-3+\sqrt{-3}}{2}$, apresentamos sua construção geométrica na Figura 11 .

Analisamos os segmentos $\overline{L P}=1$ e $\overline{L N}=3$ representam os zeros da equação $x^{2}+2 x-3=0$ (Figura 10 , isto devido ao paralelismo entre os segmentos $\overline{G J}$ e $\overline{L H}$ e o círculo com comprimento de raio igual a 2 e, posteriormente, à construção dos segmentos que são a solução do problema apresentado. Continuamos com a análise na equação $x^{2}+3 x+3=0$ (Figura 11), percebendo que não é possível a correta localização das raízes da equação, pois os segmentos $\overline{L P}=0,79$ e $\overline{L N}=3,79$ não representam as raízes da equação que pertencem ao Conjunto dos Números Complexos.

Ainda sobre a análise de todas proposições acima, inferimos que o estudante pode não somente diversificar sua prática de construção geométrica, sobretudo com o auxílio do software GeoGebra, mas também de conseguir determinar a qual conjunto numérico pertencem as soluções das equações propostas. Ressaltamos ainda que, nestas situações de estudo, nem sempre é possível determinar as soluções/raízes das equações, uma vez que os segmentos de reta, quando encontrados, não representam necessariamente as raízes das equações quadráticas. Ponderamos ainda que situações de estudo como estas, auxiliam o discente no desenvolvimento sólido do pensamento matemático, e que nestes casos o estudante tem a oportunidade de confirmar ou refutar as hipóteses advindas de sua pesquisa, propiciando maior autonomia de aprendizagem e propriedade sobre o conhecimento ora abordado. 

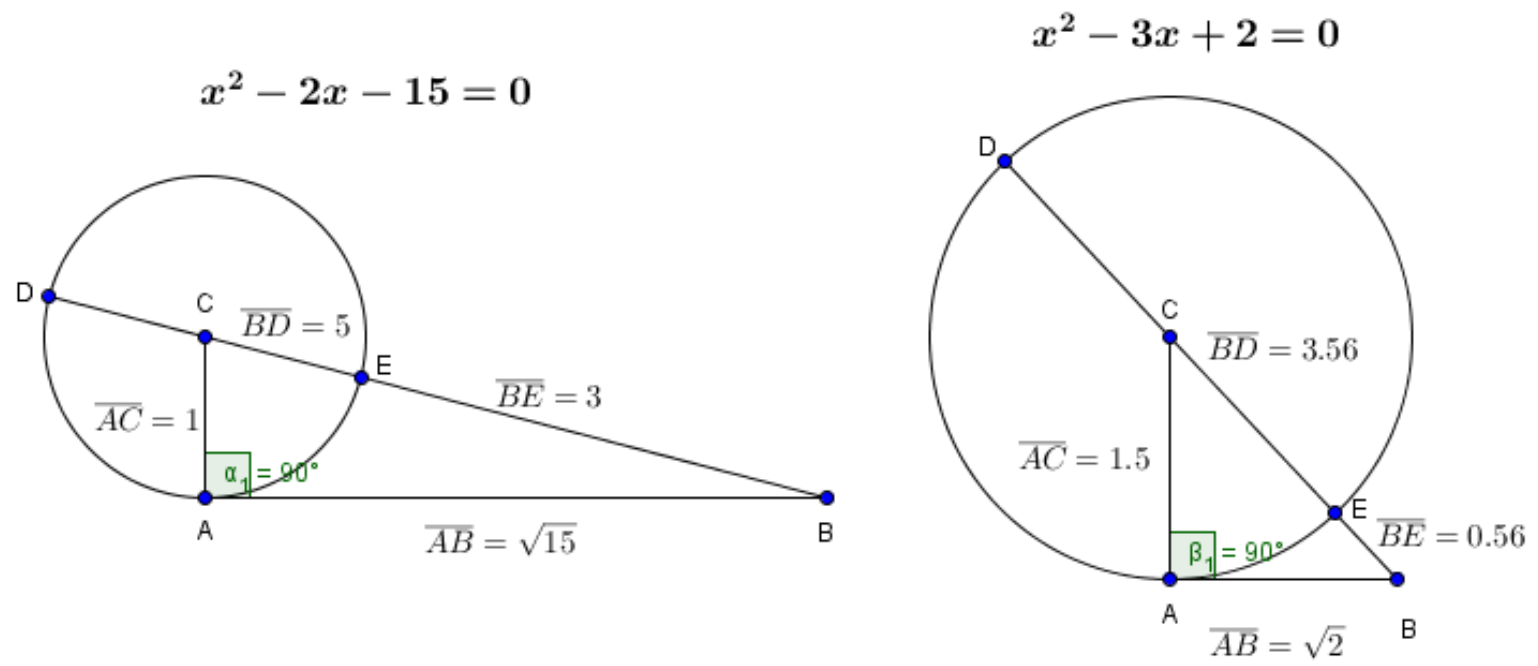

Figura 7: Resolução das equações $x^{2}-2 x-15=0$ e $x^{2}-3 x+2=0$ pelo Método de Descartes.

Fonte: Formatação Própria.

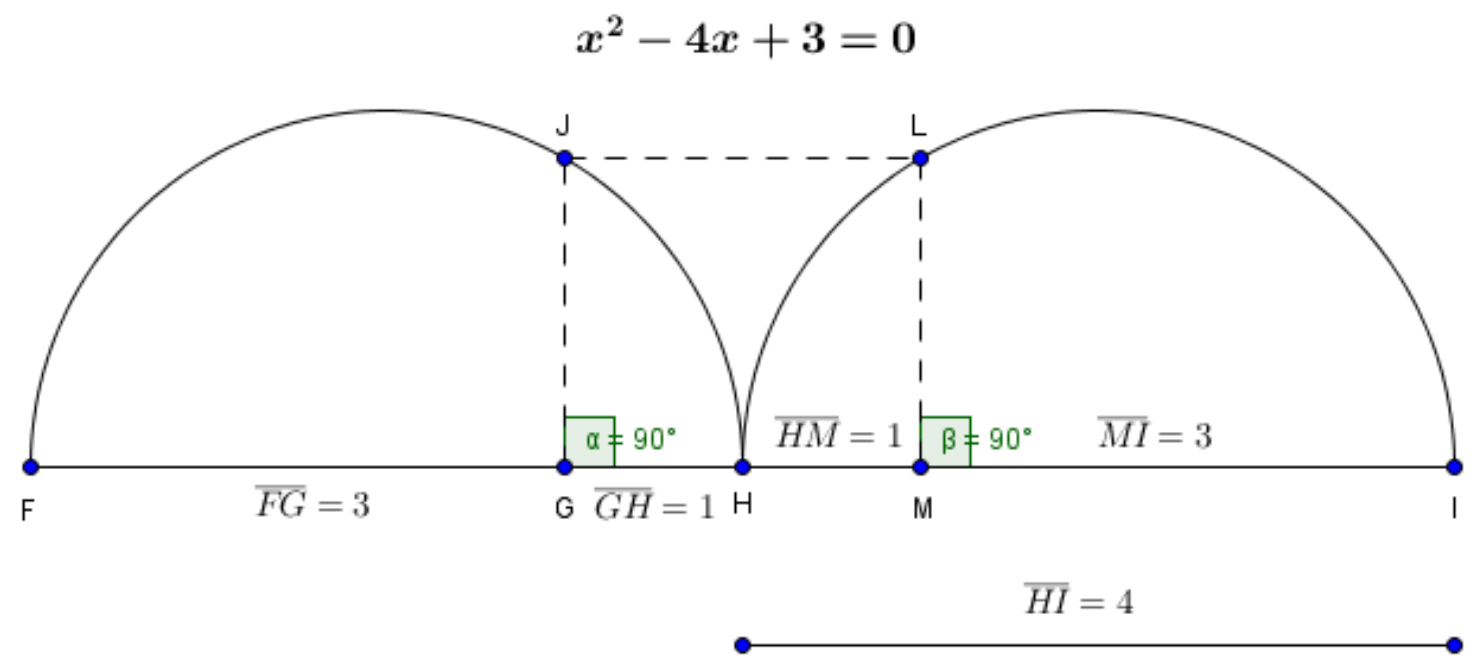

Figura 8: Resolução da equação $x^{2}-4 x+3=0$ pelo Método das Semicircunferências Tangentes.

Fonte: Formatação Própria.

Conex. Ci. e Tecnol. Fortaleza/CE, v. 10, n. 4, p. 79 - 91, dez. 2016 


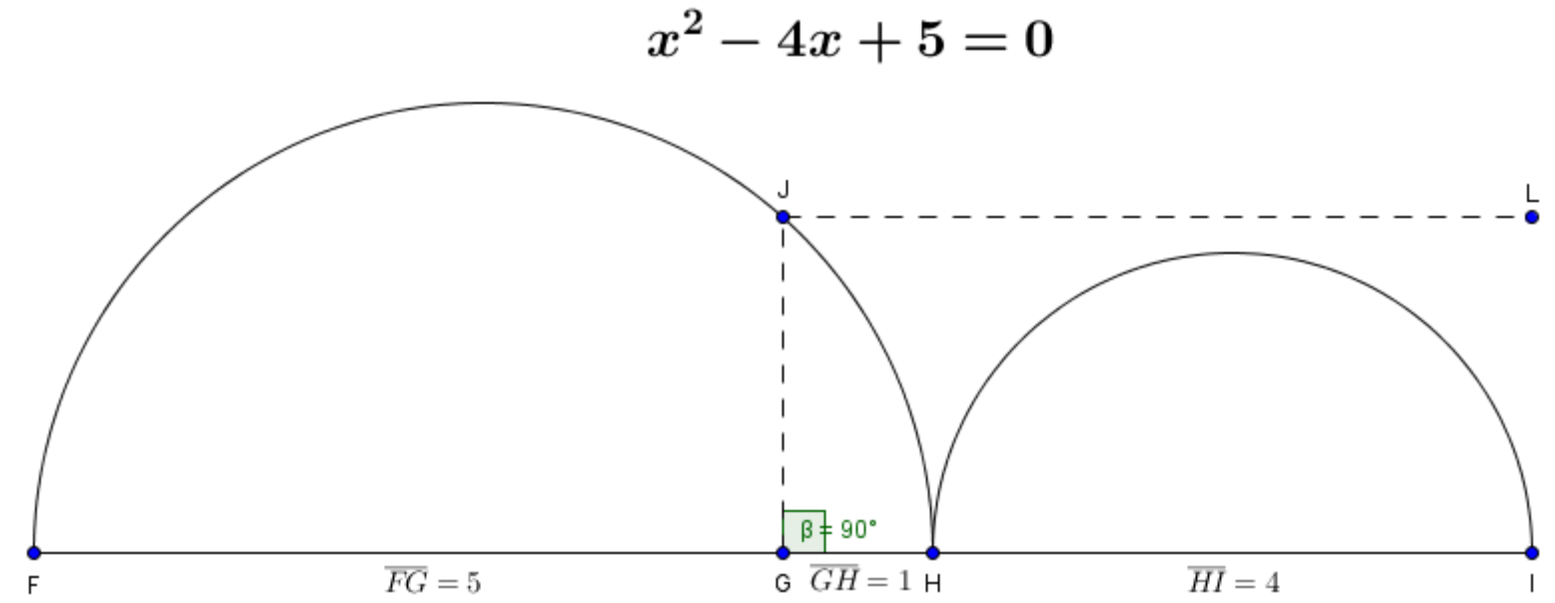

Figura 9: Resolução da equação $x^{2}-4 x+5=0$ pelo Método das Semicircunferências Tangentes

Fonte: Formatação Própria.

$$
x^{2}+2 x-3=0
$$

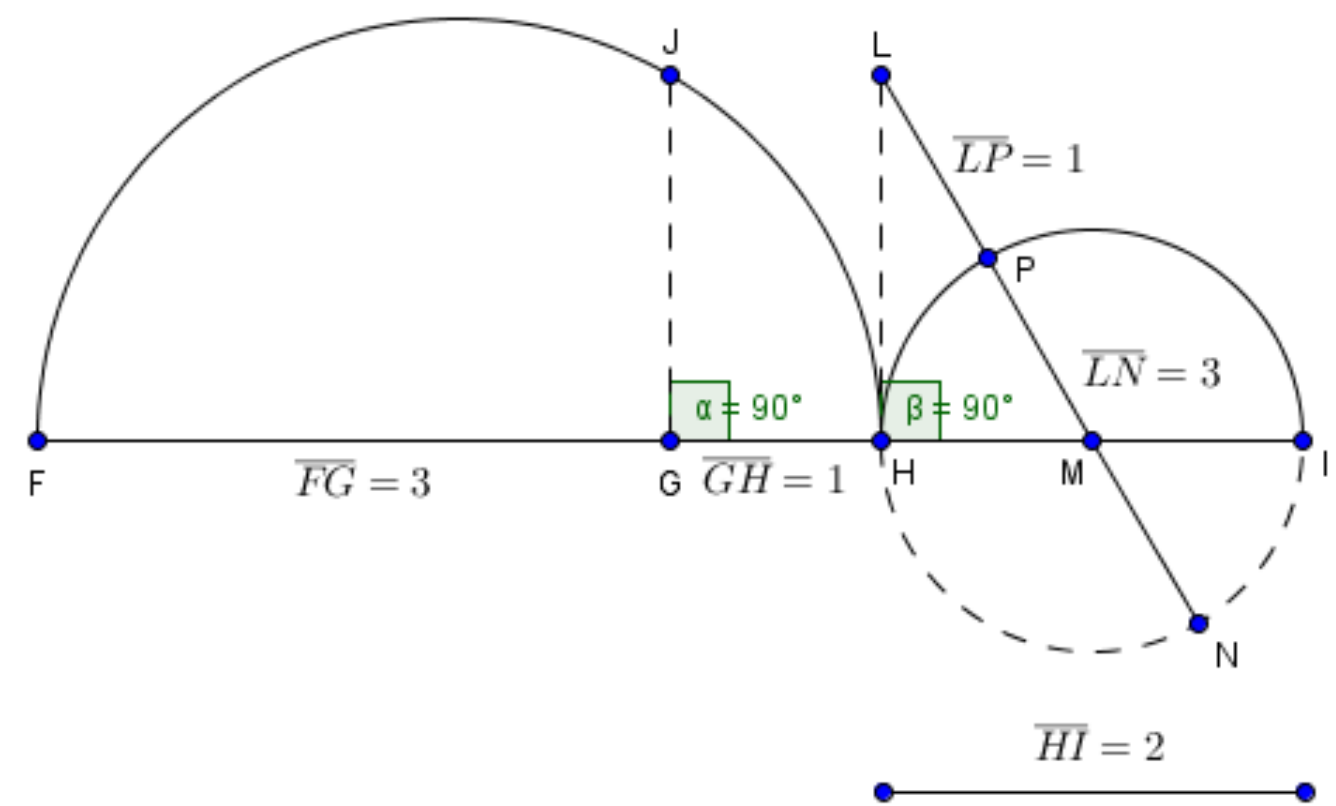

Figura 10: Resolução da equação $x^{2}+2 x-3=0$ pelo Método das Semicircunferências Tangentes.

Fonte: Formatação Própria.

Conex. Ci. e Tecnol. Fortaleza/CE, v. 10, n. 4, p. 79 - 91, dez. 2016 


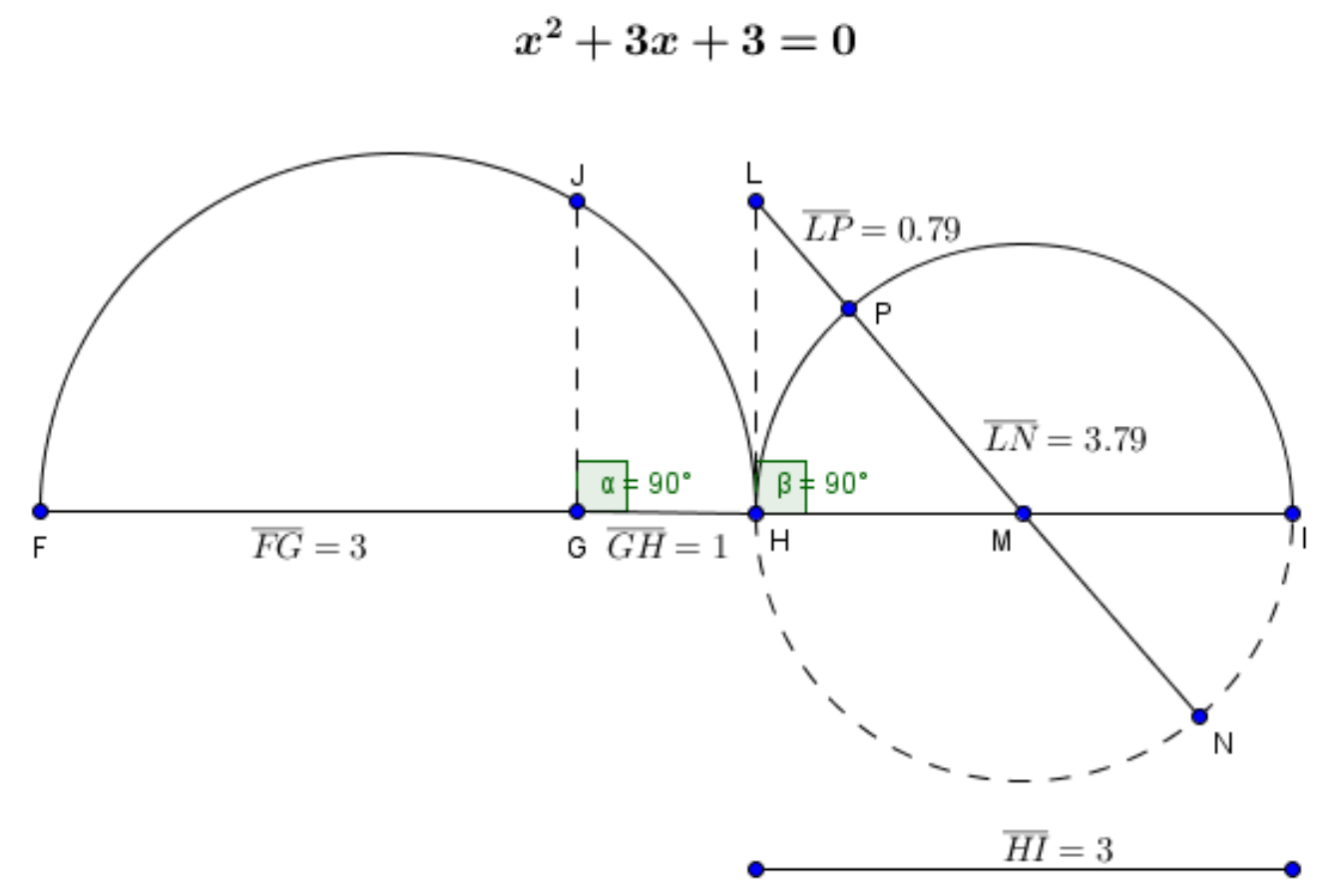

Figura 11: Resolução da equação $x^{2}+3 x+3=0$ pelo Método das Semicircunferências Tangentes.

Fonte: Formatação Própria. 


\section{CONSIDERAÇÕES FINAIS}

Este trabalho visou discutir um estudo sobre alternativas metodológicas para resolução de equações quadráticas com ênfase na perspectiva geométrica. Houve ao longo de todo o trabalho o cuidado para com a linguagem matemática, tornando-a acessível a estudantes de Matemática, sem perder, no entanto, a generalidade e o rigor necessários ao bom desenvolvimento dos estudos ora discutidos.

Nas seções passadas discorremos sobre a equação de Bhaskara, discutindo a motivação inicial e o percurso algébrico seguido pela parte significativa dos livros didáticos, com a intenção final de determinar os zeros de equações quadráticas. Propomos ainda a discussão dos modelos conhecidos como Método de Descartes - MD e Método das Semicircunferências Tangentes - MST para favorecer a discussão geométrica, como forma complementar à localização dos zeros de equações quadráticas, ressalvadas as condições restritivas de existência de cada modelo. Concluímos ainda que não somente é possível a localização exata das raízes de equações quadráticas, mas também de localizar segmentos de retas que não correspondem às referidas raízes, fato este devido à restrição dos modelos, já devidamente abordado e comentado.

Descrevemos ainda as etapas iniciais da metodologia de ensino e pesquisa em Matemática conhecida como Engenharia Didática - ED, considerando a interpretação geométrica das equações quadráticas, abordando inclusive três situações didáticas de ensino. Além disso, foram realizados estudos em literatura especializada em Matemática e em História da Matemática a fim de fundamentar os modelos geométricos resolutivos aqui propostos. Entretanto, percebemos que os mesmos não fazem parte daquelas propostas de livros didáticos, ficando a cargo apenas de literatura mais restritiva e especializada, o que não impede o uso em sala de aula com ênfase ao favorecimento da pesquisa matemática, e reiteramos ainda que este estudo pode servir de substrato para práticas no desenvolvimento do ensino de Matemática.

\section{REFERÊNCIAS}

ALMOULOUD, S. A. Fundamentos da didática da matemática. Curitiba: Ed. UFPR, 2007.

ALMOULOUD, S. A.; QUEIROZ, C. de; COUTINHO, S. Engenharia didática: características e seus usos em trabalhos apresentados no gt-19/anped. Revemat: Revista Eletrônica de Educação Matemática, v. 3, n. 1, p. 62-77, 2008.
ALVES, F. R. V. Rv \& borges neto, hermínio. a existência da sequência de fibonacci no campo dos inteiros: uma atividade de investigação apoiada nos pressupostos da sequência fedathi. Boletim Gepem, n. 59, p. 135-140, 2011. 2011(a)

Didática da matemática. Fortaleza: UAB/IFCE,

Engenharia didática para o teorema da função implícita: análises preliminares e a priori. Revista Brasileira de Ensino de Ciência e Tecnologia, v. 7, n. 3, 2014.

BOYER, C. B.; MERZBACH, U. História da matemática. $3^{\mathrm{a}}$ edição. Edição. São Paulo: Blucher, 2010.

CARNEIRO, V. C. G. Engenharia didática: um referencial para ação investigativa e para formação de professores de matemática. Zetetiké, v. 13, n. 23, p. 87-119, 2005.

CONTADOR, P. R. M. Matemática: uma breve história. : Editora Livraria da Fisica, 2008. v. 1,2.

DANTE, L. R. Matemática: ensino médio. São Paulo: Ática, v. 1,2,3, 2004.

EVES, H. W.; DOMINGUES, H. H. Introdução à história da matemática. $\sigma^{a}$ Edição. : Unicamp, 2004.

FERREIRA, G. S. S. Um breve estudo sobre equações algébricas. Imprima, Recife, 2016.

FERREIRA, G. S. S.; ALVES, F. R. V. Engenharia didática para discussão geométrica e resolução de equações de $1^{\circ}$ grau: Análises preliminares e a priori. Conexões-Ciência e Tecnologia, v. 9, n. 4, p. 78-82, 2015.

GARBI, G. G. O romance das equações algébricas. $4^{a}$ Edição. São Paulo: Editora Livraria da Física, 2010.

GIOVANNI, J. R.; BONJORNO, J. R.; JUNIOR, J.

R. G. Matemática completa. São Paulo: FTD, 2005. v. $1,2,3$.

KILHIAN, K. Resolvendo equações quadráticas pelo método de Descartes. 2012. Disponível em: $<$ http://obaricentrodamente.blogspot.com.br/2012/09/ resolvendo-equacoes-quadraticas-pelo.html> [Acesso em: 1 mar. 2015].

LORENZATO, S. Para aprender matemática. : Autores Associados, 2006. 
DISCUSSÃO GEOMÉTRICA PARA EQUAÇÕES QUADRÁTICAS E ELEMENTOS DE ENGENHARIA DIDÁTICA: ANÁLISES PRELIMINARES E A PRIORI

MARCONDES, C. A.; GENTIL, N.; GRECO, S. E.

Matemática. São Paulo: Ática, 2003. Único.

PAIVA, M. Matemática. São Paulo: Moderna, 2005.

Único.

SILVA, C. X. d.; FILHO, B. B. Matemática: aula por aula. FTD, v. 1,2,3, 2005

SMOLE, K. S.; DINIZ, M. I. Matemática: Ensino

Médio. São Paulo: Saraiva, 2005. v. 1,2,3.

TUNALA, N. Resolução geométrica da equação do $2^{\circ}$ grau. RPM - Revista do Professor de Matemática., Rio de Janeiro, v. 12, 1988. 